Annales Academiæ Scientiarum Fennicæ

Series A. I. Mathematica

Volumen 14, 1989, 133-136

\title{
A NOTE CONCERNING THE LEMMA OF JULIA-WOLFF-CARATHÉODORY
}

\author{
Maurice Heins
}

1. Let $f$ denote an analytic function mapping the open unit disk $\Delta$ into itself. An essential part of the lemma of Julia-Wolff-Carathéodory may be formulated as follows (cf. [2]):

Let $c$ denote

$$
\inf _{|z|<1} \operatorname{Re}\left(\frac{1+f(z)}{1-f(z)}\right) / \operatorname{Re}\left(\frac{1+z}{1-z}\right) .
$$

Then $(1-f(z)) /(1-z)$ possesses the sectorial limit $c^{-1}$ at 1 . Further, if $c>0$, then also $f^{\prime}$ possesses the sectorial limit $c^{-1}$ at 1 .

The object of the present note is to show that when $f$ has the Fatou limit 1 at 1 and $c=0$, it need not be the case that $\lim _{r \uparrow 1} f^{\prime}(r)$ exists. To that end we show that there exists a Blaschke product $b$ having a nonempty zero set, possessing the Fatou limit 1 at 1 , and having the property that $b^{\prime}$ has an infinite set of zeros in the open interval $(0,1)$, which clusters at 1 . By a remark of Carathéodory $[1$, p. 30$]$ we see that if $b^{\prime} \mid(0,1)$ were bounded, then $(1-b(z)) /(1-z)$ would possess a finite sectorial limit at 1 so that the associated $c$ would be positive and $\lim _{r \uparrow 1} b^{\prime}(r)>0$. This is not possible. Hence $b^{\prime} \mid(0,1)$ is not bounded. It follows that $b^{\prime}$ does not possess a radial limit at 1 .

Before turning to the construction of $b$ we make two observations.

(i) A sufficient condition for $c$ associated with $f$ to be zero is that $f$ not possess the Fatou limit 1 at 1 . Here a wide variety of possibilities lies at hand. There are trivial examples of such $f$ which are the restrictions of functions analytic at 1 (so that the limit of the derivative at 1 exists and is finite). An example of a function $f$ not possessing the Fatou limit 1 at 1 for which $\lim _{r \uparrow 1} f^{\prime}(r)$ does not exist is readily exhibited with the aid of a Blaschke product associated with the orbit containing 0 of a cyclic group of conformal automorphisms of $\Delta$ generated by a hyperbolic transformation having 1 and -1 as ixed points. It is the situation where $f$ has the Fatou limit 1 at 1 and $c=0$ which calls for attention. An example of such a function $f$ for which $f^{\prime}$ has the sectorial limit $\infty$ at 1 is the one satisfying

$$
\left(\frac{1+f(z)}{1-f(z)}\right)^{2}=\frac{1+z}{1-z}, \quad|z|<1 .
$$


(ii) In the next section a recursive procedure will be given to obtain a Blaschke product satisfying the conditions imposed on $b$. One could also use "scissors and glue" techniques to produce a concrete Riemann surface leading to an example of the desired kind. However, implicit in such an approach is the appeal to deeper resources such as the uniformization theorem. For the present purposes the use of elementary tools of classical analysis is appropriate.

2. To establish the existence of a Blaschke product $b$ having the stated properties we proceed as follows. Given $a$ real satisfying $0<|a|<1$, we introduce the Möbius transformation $M_{a}$ defined by

$$
M_{a}(z)=\frac{a+z}{1+a z} .
$$

It is clear that $M_{a}$ is hyperbolic and has fixed points -1 and 1 and that $M_{-a}$ is the inverse of $M_{a}$. We put down the sequence $\left(1-2^{-(n+1)}\right)_{0}^{\infty}$ and introduce $F_{n}$, $n \in \mathbf{N}$, defined by

$$
F_{n}(z)=M_{1-2-(n+1)}\left(z^{2}\right) .
$$

Of course, $F_{n}$ is a finite Blaschke product of degree 2. We thereupon introduce recursively a sequence $\left(G_{n}\right)_{0}^{\infty}$ of finite Blaschke products, where the degree of $G_{n}$ is $2(n+1)$ and $G_{n} \mid(-1,1)>0$, and an associated sequence $\left(\delta_{j}\right)_{0}^{\infty}$ of disjoint closed circular disks having positive radii and lying in $\Delta$ such that (a) the centers of the disks are in $\{0 \leq x<1\}$, (b) $\delta_{j+1}$ lies to the right of $\delta_{j}, j \in \mathbf{N}$, (c) for each $n \in \mathbf{N}$, for $j=0, \ldots, 2 n$, there is a zero of $G_{n}^{\prime}$ in the open circular disk having the same center as $\delta_{j}$ but half the radius, (d) $\left(G_{n}\right)_{0}^{\infty}$ is a subsequence of the sections of a convergent Blaschke product with value having Fatou limit 1 at 1 . Since the derivative of a finite Blaschke product of positive degree $m$ has $m-1$ zeros in $\Delta$ counted by multiplicity, the zeros of $G_{n}^{\prime}$ in $\Delta$ are all taken into account by the specified open subdisks of the $\delta_{j}, j=0, \ldots, 2 n$. Further, the zeros in question are simple and real.

Given $n \in \mathbf{N}$, suppose that $G_{n}$ is a finite Blaschke product of degree $2(n+1)$ such that $G_{n} \mid(-1,1)>0$ and that $\left(\delta_{j}\right)_{0}^{2 n}$ is a finite sequence of closed circular disks of positive radii, lying in $\Delta$ and having centers in $\{0 \leq x<1\}$, the disk $\delta_{0}$ being $\left\{|z| \leq 2^{-1}\right\}$. Suppose further that (i) $\delta_{j+1}$ lies to the right of $\delta_{j}$, $j=0, \ldots, 2 n-1$, when $n \geq 1$, (ii) the center of $\delta_{j}$ is to the right of $1-2^{-j}$, $j=1, \ldots, 2 n$, when $n \geq 1$, (iii) there exists a zero of $G_{n}^{\prime}$ in the open circular disk having the same center as $\delta_{j}$ but half the radius, $j=0, \ldots, 2 n$. All the zeros of $G_{n}^{\prime}$ in $\Delta$ are accounted for thereby and are simple and real. The condition (iii) is fulfilled when $n=0$ by taking $G_{0}=F_{0}$. We shall introduce (1) a finite Blaschke product $G_{n+1}$ of the form $G_{n} B_{n}$ where $B_{n}$ is a finite Blaschke product of degree 2 satisfying $B_{n} \mid(-1,1) \geq 1-2^{-(n+2)}$ and (2) disjoint closed circular disks $\delta_{2 n+k+1}, k=0,1$, of positive radii lying in $\Delta$ and having centers in $(0,1)$ 
where $\delta_{2 n+k+1}$ lies to the right of $\delta_{2 n+k}$ and the center of $\delta_{2 n+k+1}$ is to the right of $1-2^{-(2 n+k+1)}, k=0,1$, such that $G_{n+1}$ and $\left(\delta_{j}\right)_{0}^{2 n+2}$ satisfy the conditions (i), (ii), (iii) with $n$ replaced by $n+1$. As above, the zeros in question account for all the zeros of $G_{n+1}^{\prime}$ in $\Delta$ and are simple and real.

To obtain admitted $G_{n+1}, \delta_{2 n+1}, \delta_{2 n+2}$, as a first step we propose that $G_{n+1}$ be a product of the form

$$
G_{n}\left(F_{n+1} \circ M_{-t}\right)
$$

where $t \in(0,1)$ is to be further restricted. We next put down $r$ satisfying 1 $2^{-(2 n+1)}<r<1$ and the condition that $\bigcup_{0}^{2 n} \delta_{j} \subset\{|z| \leq r\}$. For $t$ sufficiently near 1 the function $G_{n+1}^{\prime}$ is uniformly close to $G_{n}^{\prime}$ on $\{|z| \leq r\}$. It follows that for $t$ sufficiently near 1 , the zeros of $G_{n+1}^{\prime}$ in $\{|z| \leq r\}$ lie in the specified open subdisks of the $\delta_{j}, j=0, \ldots, 2 n$, there is exactly one in each, the zeros in question are simple and real, and $G_{n+1} \mid(-1,1)$ has a strong relative minimum at the zero of $G_{n+1}^{\prime}$ in $\delta_{2 n}$.

We now further restrict $t$ to obtain a function $G_{n+1}$ such that $G_{n+1}^{\prime}$ has two simple zeros on the interval $(r, 1)$. The introduction of the disks $\delta_{2 n+1}, \delta_{2 n+2}$ will then be straightforward. Using (2.3) we obtain

$$
G_{n+1} \circ M_{t}=\left(G_{n} \circ M_{t}\right) F_{n+1} .
$$

We see that $\left(G_{n+1}^{\prime} \circ M_{t}\right) M_{t}^{\prime}$ tends uniformly to $F_{n+1}^{\prime}$ on $\delta_{0}$ as $t \uparrow 1$. Hence $G_{n+1}^{\prime}$ has a simple zero $\xi$ satisfying $\max \left\{1-2^{-(2 n+2)}, r\right\}<\xi<1$ at which $G_{n+1} \mid(-1,1)$ has a strong relative minimum when $t$ is sufficiently near 1 . For $t$ restricted to satisfy the above conditions $G_{n+1} \mid(-1,1)$ has a relative maximum at a point $\eta$ between the zero of $G_{n+1}^{\prime}$ in $\delta_{2 n}$ and $\xi$. We note that $G_{n+1}^{\prime}(\eta)=0$ and that $\eta>r$. We now take $\delta_{2 n+1}$ (respectively $\delta_{2 n+2}$ ) as a closed circular disk of positive radius centered at $\eta$ (respectively $\xi$ ) so that $\delta_{2 n+1}$ lies to the right of $\{|z| \leq r\}$ and $\delta_{2 n+2}$ lies to the right of $\delta_{2 n+1}$ and is contained in $\Delta$. The zeros of $G_{n+1}^{\prime} \mid \Delta$ are accounted for and are all simple and real.

The existence of allowed sequences $\left(G_{n}\right)_{0}^{\infty}$, where $G_{0}=F_{0}$, and $\left(\delta_{j}\right)_{0}^{\infty}$ follows.

Let $\delta_{j}^{*}$ denote the closed circular disk having the same center as $\delta_{j}$ and radius one half of that of $\delta_{j}, j \in \mathbf{N}$. It is now readily concluded that $\left(G_{n} \mid \Delta\right)_{0}^{\infty}$ converges to a function $b$ on $\Delta$ given by a convergent Blaschke product, that $b$ has Fatou limit 1 at 1 , that $b^{\prime}$ has a unique zero in $\delta_{j}^{*}, j \in \mathbf{N}$, and no others in $\Delta$ and that the zeros of $b^{\prime}$ are all simple and real. We remark that the parameters $t$ may be so chosen that the zeros of the resulting $b$ cluster exactly at 1 . 


\section{References}

[1] CarathÉodory, C.: Funktionentheorie II. - Verlag Birkhäuser, Basel, 1950.

[2] Nevanlinna, R.: Eindeutige analytische Funktionen, 2te Aufl. - Springer-Verlag, Berlin, 1953.

University of Maryland

Department of Mathematics

College Park, MD 20742

U.S.A.

Received 8 March 1988 